\title{
Comparison of the currents in the Dirichlet and Neumann shortwave diffraction problems of a plane wave from smooth prolate bodies of revolution
}

\author{
Anna Kirpichnikova \\ Department of Computing Science and Mathematics, University of Stirling, Scotland UK; \\ e-mail: anya@cs.stir.ac.uk \\ Natalia Kirpichnikova \\ Saint-Petersburg Department of Steklov's Mathematical Institute, Russia; \\ e-mail: nkirp@pdmi.ras.ru
}

\begin{abstract}
This paper continues the series of works [1-5] on the shortwave diffraction on the prolate body of revolution. The numerical comparison of the wave currents for Dirichlet and Neumann boundary conditions confirms the continuous transition of the current from the lit area into the shadowed zones through Fock's zone. The formulae for the currents were obtained according to the LeontovichFock parabolic equation method [6]. We investigated the influence of the correction term that contains the large parameter, on the Fock's current. This large parameter reflects body's elongation. Diffraction formulae obtained in $[1,4]$, give the integral representation of the field in some neighborhood of the point, which is located on the boundary of geometric shadow. These formulae give a continuous transformation from ray field in the lit area to the field in the shadow using Fock's currents.
\end{abstract}

\section{INTRODUCTION}

This paper considers an axi-symmetric shortwave diffraction problem of a plane wave. The plane wave incidents a strictly convex prolate body of revolution $\Omega$ along the rotation axis. The prolate body $\Omega$ (obstacle, scatterer) has two major geometric characteristics at its surface $\partial \Omega$ : curvature radii along and perpendicular to the rotation axis. Both radii are assumed to be much larger than the wavelength of the incident wave. Wave field $U$ satisfies Helmholtz equation $\left(\Delta+k^{2}\right) U=0$, where $k \gg 1$ is the wave-number. Either the Dirichlet or the Neumann condition is set on the surface of the scatterer. We choose a solution that satisfies the limiting absorption principle at far distances from the body.

The Equator coincides with the shadow-light boundary created by the plane incident wave. We denote by $s$ the arc length along the geodesic curve on the surface of revolution, measured from the Equator, and $n$ is the coordinate along the outward normal to the scatterer's surface. It measures the distance from $\partial \Omega$ to the observation point $(s, n)$. We find the axi-symmetric solution of the problem, namely the wave filed $U$, such that

$$
\frac{\partial U}{\partial \varphi}=0, \quad 0 \leq \varphi \leq 2 \pi
$$

where $\varphi$ is the angle of body revolution.

The solution has the form $U=e^{i k s} W(s, n)$, where $e^{i k s}$ describes the main oscillations of the wave field, and $W$ is the attenuation function.

We use the Fock-Leontovich parabolic equation method $[1,4,6]$ to obtain the first three terms of the asymptotic expansion

$$
U=e^{i k s} W=e^{i k s}\left(W_{0}+\frac{W_{1}}{k^{\frac{1}{3}}}+\frac{W_{2}}{k^{\frac{2}{3}}}+\mathcal{O}\left(k^{-1}\right)\right),
$$

where each of the terms $W_{j}=W_{j}^{\text {inc }}+W_{j}^{\text {ref }}, j=$ $0,1,2$, is the full wave field, namely the sum of the incident and the reflected waves. We call $W_{0}$ the main, $W_{1}$ the first correcting and $W_{2}$ the second correcting terms of the expansion. Expansions (1)

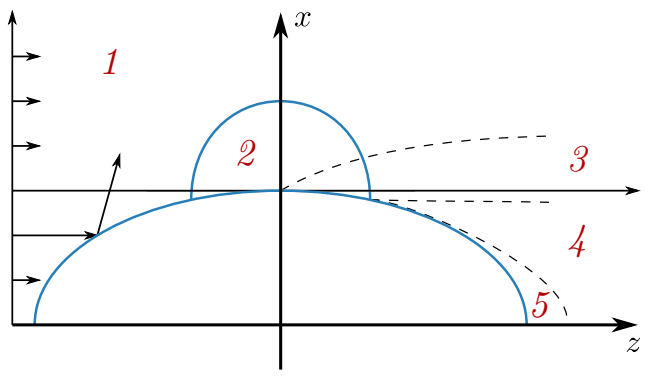

Figure 1: The main five zones near $\partial \Omega$ : lit zone 1, the Fock's zone 2, neighborhood of the limit ray 3 , zone 4 is a shadow, zone 5 is a surface layer full of creeping waves. 


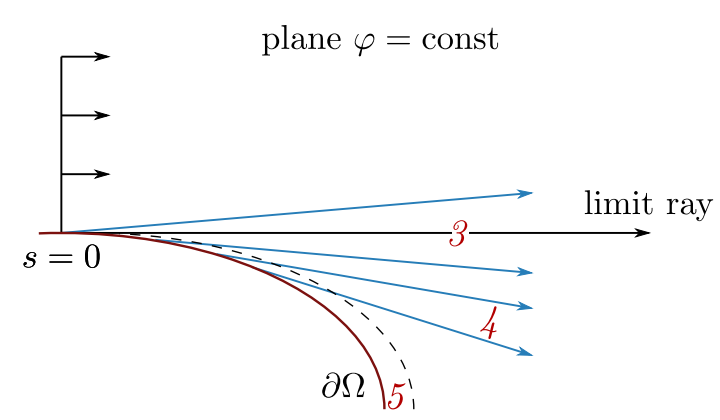

Figure 2: Penumbra and shadow regions $\sigma>0$. The field in the shadow region 4 between regions 3 and 5 has ray nature of the leaving wave. Region 5 is the creeping waves zone with $\nu=\mathcal{O}(1), s=\mathcal{O}(1)$.

have been obtained for both the Dirichlet [1] and the Neumann problems [4].

The field has been constructed in the vicinity of the light-shadow boundary, see zone 3 in Fig. 1, this is a penumbra Fock's region. The point with coordinates $(s=0, n=0)$ belongs to the geometric boundary of the shadow, which is the Equator. In the neighborhood of that point, we introduce Fock's boundary layer, where $\mathcal{O}\left(k^{\frac{1}{3}} s\right)=\mathcal{O}(1)$, $\mathcal{O}\left(k^{\frac{2}{3}} n\right)=\mathcal{O}(1)$. Body $\Omega$ is three-dimensional, we use coordinates $(s, n, \varphi)$ in the vicinity of the Equator, see Fig. 2. Since we measure the distance $s$ from the Equator, $s=0$ at the Equator; $s>0$ corresponds to the shadowed part of the surface; $s<0$ corresponds to the lit zone. Fock's zone was called "field nucleus" by V. M. Babich [7]. If we know the wave field in this zone, we can construct an asymptotic of the wave field in the lit region 1 , in the vicinity of the extreme ray 3 and in the shadowed region 4 . In our case, this zone is a circular domain in the vicinity of the light-shadow boundary (near the Equator), where coordinates $s$ and $n$ are quite small. Thus we use the stretched dimensionless coordinates $(\sigma, \nu)$ as

$$
\sigma=\frac{M_{0}}{\rho_{0}} s, \quad \nu=\frac{2 M_{0}^{2}}{\rho_{0}} n .
$$

Here $\boldsymbol{M}_{0}$ is the Fock's large parameter, such that formula (2) defines $\rho_{0}$ as the curvature radius of geodesics (meridians) on the Equator:

$$
\left(\frac{k \rho_{0}}{2}\right)^{\frac{1}{3}}=\boldsymbol{M}_{0}, \quad \rho_{0}=\left.\rho(s)\right|_{s=0} .
$$

The aforementioned formulae are valid in any plane $\varphi=$ const, for instance, when $\varphi=0$.

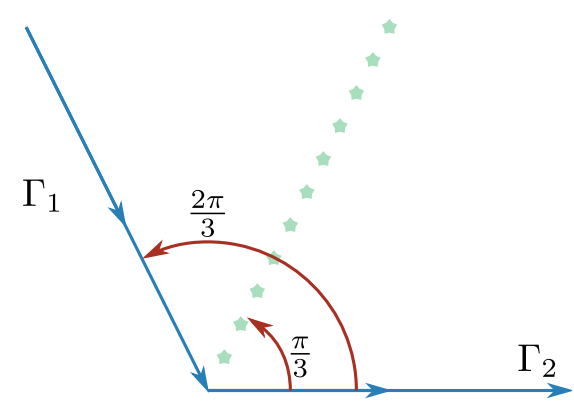

Figure 3: Integration contour $\Gamma=\Gamma_{1} \cup \Gamma_{2}$ goes along the straight line from $\infty e^{i \frac{2 \pi}{3}}$ to 0 , and then from 0 to $\infty$ along the positive real axis in the complex plane of $\zeta$. The roots of $w_{1}(\zeta)$ and $w_{1}^{\prime}(\zeta)$ belong to the ray $e^{i \frac{\pi}{3}}$ (shown as stars).

2 The CURRENTS FOR THE MAIN TERM $W_{0}$ IN THE PENUMBRA ZONE

We follow V. A. Fock [6] and present the main term of asymptotics (1) in a form of a contour integral for the Dirichlet problem:

$W_{0}^{\text {Dir }}=\frac{1}{\sqrt{\pi}} \int_{\Gamma} e^{i \sigma \zeta}\left[v(\zeta-\nu)-\frac{v(\zeta)}{w_{1}(\zeta)} w_{1}(\zeta-\nu)\right] d \zeta$

and for the Neumann problem:

$W_{0}^{\mathrm{Neu}}=\frac{1}{\sqrt{\pi}} \int_{\Gamma} e^{i \sigma \zeta}\left[v(\zeta-\nu)-\frac{v^{\prime}(\zeta)}{w_{1}^{\prime}(\zeta)} w_{1}(\zeta-\nu)\right] d \zeta$

Contour $\Gamma$ is described in Fig. 3. This contour covers the roots of Airy function $w_{1}\left(\zeta_{q}\right), q=1,2, \ldots$, in the first quadrant, or the roots of its derivative $w_{1}^{\prime}\left(\zeta_{q}^{\prime}\right)$. The roots of Airy function $w_{1}(\zeta)$ and its derivative belong to the ray $e^{i \frac{\pi}{3}}$.

The main approximation of the Dirichlet problem current $I_{0}^{\text {Dir }}$ is a value of the derivative of $W_{0}^{\text {Dir }}$ with respect to the normal $n$ at $n=0$, i.e.,

$$
I_{0}^{\text {Dir }}=\left.\frac{\partial}{\partial n} W_{0}^{\text {Dir }}\right|_{n=0}=\left.\frac{\partial}{\partial \nu} W_{0}^{\text {Dir }} \cdot \frac{\partial \nu}{\partial n}\right|_{n=0}
$$

or, going further, since the Wronskian is $v(\zeta) w_{1}^{\prime}(\zeta)-v^{\prime}(\zeta) w_{1}(\zeta)=1$

$$
I_{0}^{\mathrm{Dir}}=\frac{2 \boldsymbol{M}_{0}^{2}}{\rho_{0}} \frac{1}{\sqrt{\pi}} \int_{\Gamma} \frac{e^{i \sigma \zeta} d \zeta}{w_{1}(\zeta)} .
$$

Thus the dimensionless Dirichlet current for the numerical implementation is

$$
k^{-1} I_{0}^{\text {Dir }}=\frac{1}{M_{0}} \frac{1}{\sqrt{\pi}} \int_{\Gamma} \frac{e^{i \sigma \zeta} d \zeta}{w_{1}(\zeta)}=\frac{1}{\boldsymbol{M}_{0}} f(\sigma) .
$$


For the Neumann problem, the main approximation to the current is the value of the full field $W_{0}^{\mathrm{Neu}}$ on the surface of the scatterer, i.e., when $n=0$ :

$$
I_{0}^{\mathrm{Neu}}=\left.W_{0}^{\mathrm{Neu}}\right|_{n=0}=\frac{1}{\sqrt{\pi}} \int_{\Gamma} e^{i \sigma \zeta} \frac{d \zeta}{w_{1}^{\prime}(\zeta)}=g(\sigma) .
$$

In the book [6] by V. A. Fock, the currents introduced for the Dirichlet problem $f(\sigma)$, and for the Neumann problem, $g(\sigma)$, have the following forms

$$
\begin{aligned}
& f(\sigma)=\frac{1}{\sqrt{\pi}} \int_{\Gamma} e^{i \sigma \zeta} \frac{d \zeta}{w_{1}(\zeta)}, \\
& g(\sigma)=\frac{1}{\sqrt{\pi}} \int_{\Gamma} e^{i \sigma \zeta} \frac{d \zeta}{w_{1}^{\prime}(\zeta)} .
\end{aligned}
$$

Together with integrals $(7),(8)$, V. A. Fock introduced functions $F(\sigma), G(\sigma)$, corresponding to the Dirichlet and Neumann problems:

$$
F(\sigma)=e^{\frac{i \sigma^{3}}{3}} f(\sigma), \quad G(\sigma)=e^{\frac{i \sigma^{3}}{3}} g(\sigma) .
$$

Then the dimensionless current for the Dirichlet and Neumann problem is equal in the main approximation to

$$
\begin{aligned}
k^{-1} I_{0}^{\mathrm{Dir}} & =\frac{1}{M_{0}} e^{-i \frac{\sigma^{3}}{3}} F(\sigma), \\
I_{0}^{\mathrm{Neu}} & =e^{-i \frac{\sigma^{3}}{3}} G(\sigma) .
\end{aligned}
$$

3 Comparison of the CURRENTS IN MAIN APPROXIMATION WITH RAY SOLUTION

We compare Fock's currents (5), (6) (or (9), (10)) with the ray solution in the lit area 1 , Fig. 1. V.A. Fock showed that with $\sigma$ large and negative, the reflected field coincides with the reflected geometric-optical wave in the Fock's boundary layer

$$
\begin{array}{r}
W^{\mathrm{ref}}=\mp \sqrt{\frac{\left|\sigma_{*}\right|}{\sqrt{\sigma^{2}+3 \nu}}} \exp \left[i \left(-\frac{5}{27} \sigma^{3}-\frac{\nu \sigma}{3}\right.\right. \\
\left.\left.+\frac{4}{27}\left(\sigma^{2}+3 \nu\right)^{\frac{3}{2}}\right)\right],
\end{array}
$$

where $\sigma_{*}=\frac{2}{3} \sigma-\frac{1}{3} \sqrt{\sigma^{2}+3 \nu}, \sigma=-|\sigma|<0$ (see [3]). The minus sign in front of a radical corresponds to the Dirichlet, and the plus sign to the Neumann boundary condition. As $\sigma \rightarrow-\infty$ and restricting $\nu=\mathcal{O}(1)$, formula (11) can be simplified. Denoting the expression for the reflected wave by $W_{-\infty}^{\text {ref }}$, we write

$$
W_{-\infty}^{\mathrm{ref}}=\mp \frac{\sqrt{2 \sigma^{2}+\nu}}{\sqrt{2 \sigma^{2}+3 \nu}} e^{-i\left(\nu \sigma+\frac{\sigma^{3}}{3}\right)} .
$$

The full field in the lit zone as $\sigma \rightarrow-\infty$ is then equal to

$$
\begin{aligned}
W^{\mathrm{ray}} & =W^{\mathrm{inc}}+W_{-\infty}^{\mathrm{ref}} \\
& =e^{i\left(\nu \sigma-\frac{\sigma^{3}}{3}\right)} \mp \frac{\sqrt{2 \sigma^{2}+\nu}}{\sqrt{2 \sigma^{2}+3 \nu}} e^{-i\left(\nu \sigma+\frac{\sigma^{3}}{3}\right)} .
\end{aligned}
$$

Then the dimensionless current for the Dirichlet problem has the following form as $\sigma \rightarrow-\infty$ :

$$
k^{-1} I_{0}^{\text {Dir }}=\frac{1}{\boldsymbol{M}_{0}}\left(2 i \sigma+\mathcal{O}\left(\sigma^{-2}\right)\right) e^{-i \frac{\sigma^{3}}{3}} .
$$

Comparing formulae (9) and (12), we get

$$
F(\sigma)=2 i \sigma+\mathcal{O}\left(\sigma^{-2}\right), \text { as } \sigma \rightarrow-\infty .
$$

Similarly, for the Neumann problem, the current is equal to

$$
I_{0}^{\mathrm{Neu}}=\left[2+\mathcal{O}\left(\sigma^{-3}\right)\right] e^{-i \frac{\sigma^{3}}{3}}, \quad \text { as } \quad \sigma \rightarrow-\infty .
$$

Formulae (10), (13) imply that

$$
G(\sigma)=2+\mathcal{O}\left(\sigma^{-3}\right), \text { as } \sigma \rightarrow-\infty .
$$

Under Ray method assumptions, the current on the perfectly reflecting boundary $(s<0)$ equals the double of the current of the reflected wave. Moreover, the current is vanishing in the penumbra $(s>0)$.

\section{Currents in the Shadowed ZONE}

The incident waves do not penetrate the penumbra region $(s>0)$, but the integrand $\exp (i \sigma \zeta)$ of $(5)$, (6), decays exponentially at $\sigma>0$ in the upper halfplane $(\operatorname{Im} \zeta>0)$, since $|\exp (i \sigma \zeta)|=\exp (-\sigma \operatorname{Im} \zeta)$. In this case the integration contour $\Gamma$ should be lifted up into the upper half-plane. The poles of the integrand, which are the roots of either Airy function $w_{1}\left(\zeta_{q}\right)=0, q=1,2, \ldots$, or the roots of the derivative of Airy function $w_{1}^{\prime}\left(\zeta_{q}^{\prime}\right)=0$, become residues under the latter contour shift. The roots belong to the ray $\arg (\zeta)=\frac{\pi}{3}$. In the shadow zone, functions $F(\sigma), G(\sigma), \sigma>0$, decay exponentially as $\exp \left(-\sigma \operatorname{Im} \zeta_{q}\right), q=1,2, \ldots$, or $\exp \left(-\sigma \operatorname{Im} \zeta_{q}^{\prime}\right)$. Therefore, functions $F(\sigma)$ and $G(\sigma)$ describe gradual decay of the field amplitude on the way from the light to the shadow.

In the shadowed part of the scatterer, we consider the main term of the Dirichlet asymptotics (3) as $\sigma>0$, recalling that $\zeta_{q}$ are the roots of Airy 
function, $w_{1}\left(\zeta_{q}\right)=0$. Thus, the residue at pole $\zeta_{q}$, $q=1,2, \ldots$, for the integral (3) equals to

$$
\operatorname{Res}_{\zeta \rightarrow \zeta_{1}} W_{0}^{\text {Dir }}=-2 \sqrt{\pi} i \sum_{q=1} \frac{w_{1}\left(\zeta_{q}-\nu\right)}{\left[w_{1}^{\prime}\left(\zeta_{q}\right)\right]^{2}} e^{i \sigma \zeta_{q}} .
$$

Then, the dimensionless current $k^{-1} I_{0}^{\text {Dir }}$ for the Dirichlet problem equals to the sum of exponentially decaying currents

$$
\begin{aligned}
k^{-1} I_{0}^{\mathrm{Dir}} & =\sum_{q=1} \frac{1}{\boldsymbol{M}_{0}} \frac{2 \sqrt{\pi} i}{w_{1}^{\prime}\left(\zeta_{q}\right)} e^{\frac{\sigma\left|\zeta_{q}\right|}{2}(i-\sqrt{3})} \\
& =\frac{1}{\boldsymbol{M}_{0}} e^{-i \frac{\sigma^{3}}{3}} F(\sigma) .
\end{aligned}
$$

It is enough to compute function $F(\sigma)$ given by (7) in the shadow zone for $q=1$, since $\left|\zeta_{q}\right|>\left|\zeta_{1}\right|$ for all $q>1$, thus the corresponding terms decay faster than $\exp \left(-\sigma\left|\zeta_{1}\right| \frac{\sqrt{3}}{2}\right)$. In the shadow zone, function

$$
F(\sigma) \approx e^{i\left(\frac{\sigma^{3}}{3}+\frac{\sigma\left|\zeta_{1}\right|}{2}\right)} c_{1} \cdot e^{-\sigma\left|\zeta_{1}\right| \frac{\sqrt{3}}{2}}
$$

decays exponentially, i.e., amplitude decreases as we go deeper into shadow.

Thus, for the Neumann case and (4), we have

$$
\operatorname{Res}_{\zeta \rightarrow \zeta_{q}^{\prime}} W_{0}^{\mathrm{Neu}}=2 \sqrt{\pi} i \sum_{q=1} \frac{w_{1}\left(\zeta_{q}^{\prime}-\nu\right)}{\zeta_{q}^{\prime}\left[w_{1}\left(\zeta_{q}^{\prime}\right)\right]^{2}} e^{i \sigma \zeta_{q}^{\prime}} .
$$

The Neumann dimensionless current also equals the sum of the currents

$$
\begin{aligned}
I_{0}^{\mathrm{Neu}} & =e^{\frac{-i \sigma^{3}}{3}} G(\sigma) \\
& =\sum_{q=1} 2 \sqrt{\pi} i e^{\frac{\sigma\left|\zeta_{q}^{\prime}\right|}{2}(i-\sqrt{3})} \frac{1}{\zeta_{q}^{\prime} w_{1}\left(\zeta_{q}^{\prime}\right)} .
\end{aligned}
$$

In the shadow zone, function $G(\sigma)$ also decays exponentially,

$$
G(\sigma) \approx c_{2} e^{i\left(\frac{\sigma^{3}}{3}+\frac{\sigma\left|\zeta_{1}^{\prime}\right|}{2}\right)} e^{-\sigma\left|\zeta_{1}^{\prime}\right| \frac{\sqrt{3}}{2}} .
$$

Thus, due to the presence of the negative exponent in $F(\sigma)$ and $G(\sigma)$ in the shadow zone, the current in the shadow decays fast. Fock's formulae (3), (4) give continuous transition of the current from the lit zone to the shadow zone in both Dirichlet and Neumann problems [6]. Table below present the first three roots of the Airy function $w_{1}\left(\zeta_{q}\right)=0$ and its derivative $w_{1}^{\prime}\left(\zeta_{q}^{\prime}\right)=0$ :

$$
\begin{aligned}
\zeta_{1} & =2.33811 \cdot e^{i \frac{\pi}{3}}, & \zeta_{1}^{\prime} & =1.01879 \cdot e^{i \frac{\pi}{3}}, \\
\zeta_{2} & =4.08795 \cdot e^{i \frac{\pi}{3}}, & & \zeta_{2}^{\prime}=3.24820 \cdot e^{i \frac{\pi}{3}}, \\
\zeta_{3} & =5.52056 \cdot e^{i \frac{\pi}{3}}, & \zeta_{3}^{\prime} & =4.82010 \cdot e^{i \frac{\pi}{3}} .
\end{aligned}
$$

Even though the currents are valid near the surface, they can be used to calculate the field at far distances also by using quadratures.

\section{Correction terms $W_{2}$}

Consider the correction terms $W_{1}$ and $W_{2}$ in (1). Calculation of these terms requires time and effort $[1,4]$. The second large parameter $\boldsymbol{\Lambda}_{0}=\frac{\rho_{0}}{f(0)}$ appears to matter due to the shape of the scatterer, this parameter measures its prolongation. Here $f(0)$ is the curvature radius of the Equator.

For the numerical experiment we take $\rho_{0}^{\prime}=0$. Then the first correction term $W_{1}$ vanishes, as $\rho_{0}^{\prime}$ is its factor. Prolongation parameter $\boldsymbol{\Lambda}_{0}$ appears in the second correction term $W_{2}$ in combination with $\boldsymbol{M}_{0}$, namely, as $\frac{\boldsymbol{\Lambda}_{0}}{2 \boldsymbol{M}_{0}^{2}}$ and their positive powers. The restriction of validity of the obtained asymptotic series then is $\boldsymbol{\Lambda}_{0}=2 \boldsymbol{M}_{0}^{2-\varepsilon}, 0<\varepsilon<2$. When $\varepsilon=2$, we have $\boldsymbol{\Lambda}_{0}=\mathcal{O}(1)$ and both curvatures' radii does not differ much from each other, which is not a case of prolate body.

In the case of $\varepsilon=0$, the large parameter $\boldsymbol{\Lambda}_{0}$ compensates the Fock's large parameter $\boldsymbol{M}_{0}$. Therefore, the system of equations

$$
\begin{gathered}
\mathcal{L}_{0} W_{0}=0 \\
\mathcal{L}_{0} W_{1}+\mathcal{L}_{1} W_{0}=0 \\
\mathcal{L}_{0} W_{2}+\mathcal{L}_{1} W_{1}+\mathcal{L}_{2} W_{0}=0
\end{gathered}
$$

loses its asymptotic nature and all the equations get singular in their coefficients [2].

The current from the correction term $W_{2}$ in the Dirichlet problem has the following form on the surface $\partial \Omega[1]$

$$
\begin{aligned}
& k^{-1} I^{\text {Dir }}=\frac{1}{\boldsymbol{M}_{0} \sqrt{\pi}} \int_{\Gamma} \frac{e^{i \sigma \zeta}}{w_{1}(\zeta)} d \zeta \\
& +\frac{\boldsymbol{\Lambda}_{0}}{2 \sqrt{\pi} \boldsymbol{M}_{0}^{3}} \int_{\Gamma} e^{i \sigma \zeta}\left[\frac{\zeta}{w_{1}(\zeta)}-\frac{\left[w_{1}^{\prime}(\zeta)\right]^{2}}{\left[w_{1}(\zeta)\right]^{3}}\right] d \zeta .
\end{aligned}
$$

The dimensionless variable $\sigma$ describes the position of the observation in the Fock's zone in (14). The corresponding current for the Neumann case is then as follows:

$$
\begin{aligned}
& I^{\mathrm{Neu}}=\frac{1}{\sqrt{\pi}} \int_{\Gamma} \frac{e^{i \sigma \zeta}}{w_{1}^{\prime}(\zeta)} d \zeta \\
& \quad+\frac{1}{\sqrt{\pi}} \frac{\boldsymbol{\Lambda}_{0}}{2 \boldsymbol{M}_{0}^{2}} \int_{\Gamma} e^{i \sigma \zeta}\left[\frac{\zeta}{w_{1}^{\prime}(\zeta)}-\frac{\zeta^{2}\left[w_{1}(\zeta)\right]^{2}}{\left[w_{1}^{\prime}(\zeta)\right]^{3}}\right] d \zeta
\end{aligned}
$$



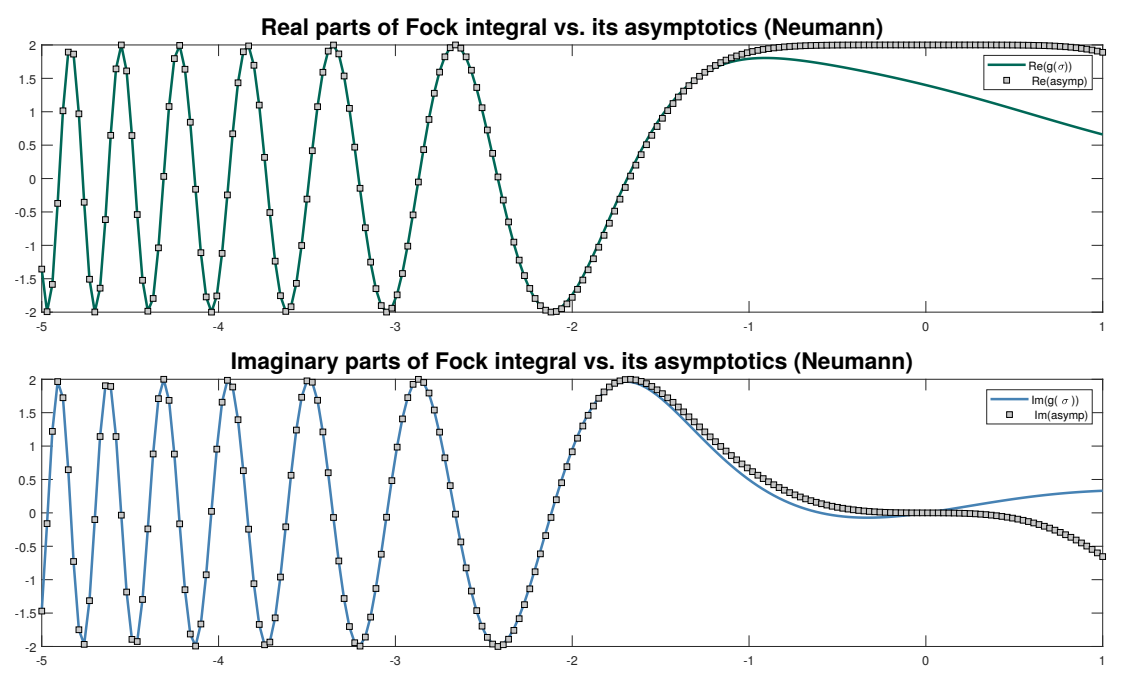

Figure 4: Neumann Fock's current (6) and its asymptotics (13) in the lit zone and the Fock's zone $(-5 \leq \sigma \leq 1)$.
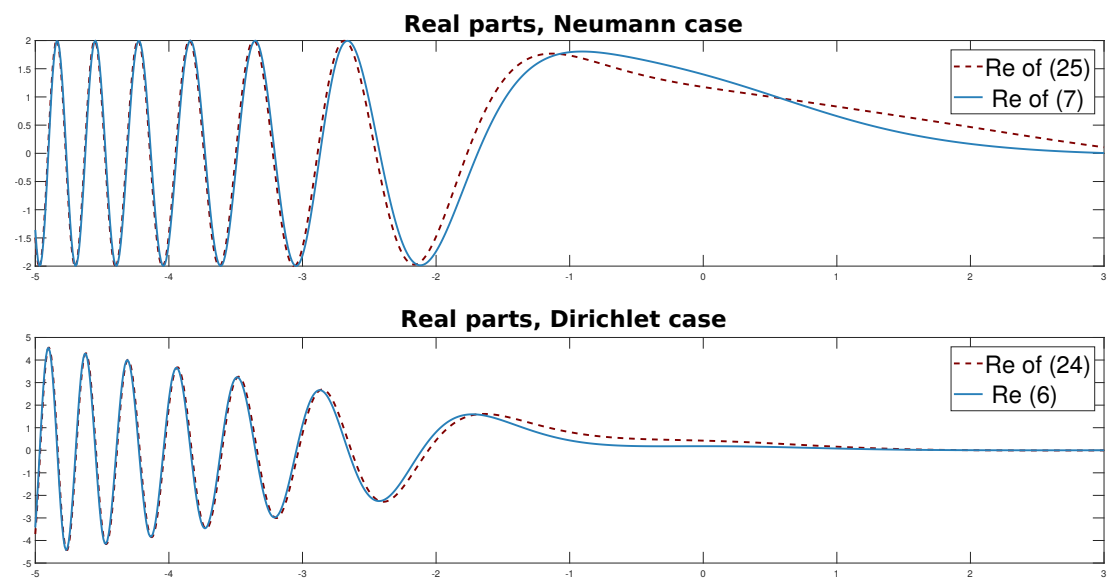

Figure 5: Real parts of Fock's currents (6), (5), are shown with solid line; and the currents with the correction terms (15), (14), are shown with dashed line; here $\varepsilon=0.1, k \rho_{0}=20$, $-5 \leq \sigma \leq 3$.

\section{NumERICAL RESUlTS}

To compare the ray current vs Fock's current (6) with its asymptotics (13) for the Neumann problem, we have chosen $k \rho_{0}=20, M_{0}=10^{\frac{1}{3}}$, namely $\frac{\boldsymbol{\Lambda}_{0}}{2 \boldsymbol{M}_{0}^{2}}=\frac{1}{\boldsymbol{M}_{0}^{\varepsilon}}$ and $k \rho_{0}=50, \boldsymbol{M}_{0}=25^{\frac{1}{3}}$. As we are interested in the influence of body elongation parameters, we will compare two cases of $\varepsilon=0.1$ which correspond to the case of prolate body, and $\varepsilon=1.5$, which corresponds to the case of a nearly spherical body. We have also computed the currents for $k \rho_{0}=50$, however, the character of the curves is the same as for $k \rho_{0}=20$, hence we do not present these results here.
The choice has been made to compare the outputs with the results in [5]. The horizontal axis covers the lit area, namely $-5 \leq \sigma \leq 1$, step $h=0.05$. The vertical axis is real and imaginary parts of the dimensionless currents, see Fig. 4.

To present the influence of the large parameters that describe the prolongation of the body, we plot formulae (15) and Fock's current (6), (8). The current coincides with the current obtained by the ray method in the Fock's zone, see Figs. 5-8.

The correction term for the Dirichlet case does not affect the field as the influence of the prolongation appears to be very small. The current coincides with the current obtained by the ray method 

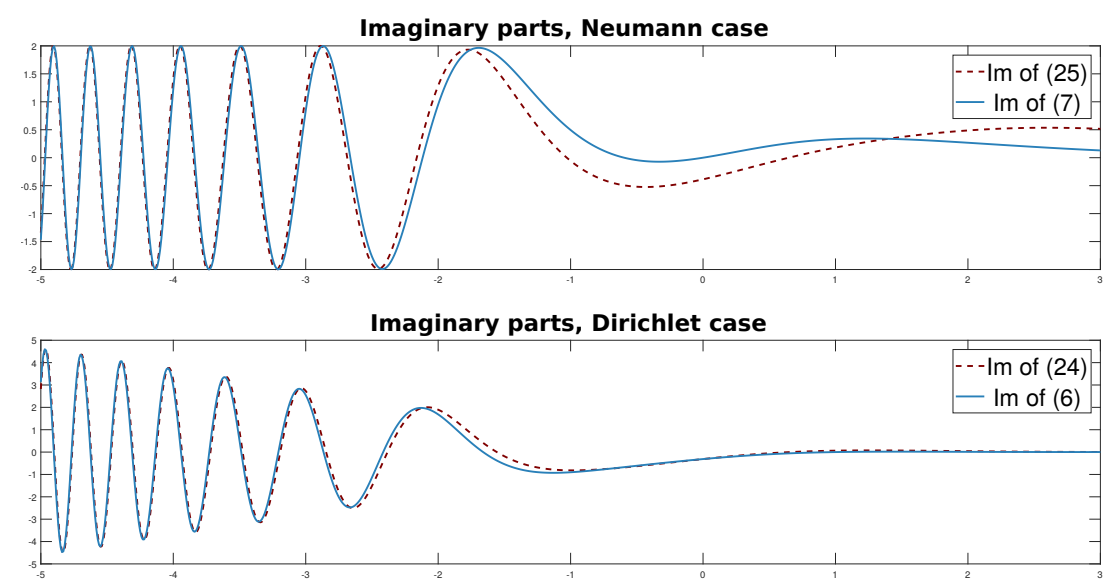

Figure 6: Imaginary parts of Fock's currents (6), (5), are shown with solid line; and the currents with the correction terms (15), (14), are shown with dashed line; here $\varepsilon=0.1$, $k \rho_{0}=20,-5 \leq \sigma \leq 3$.
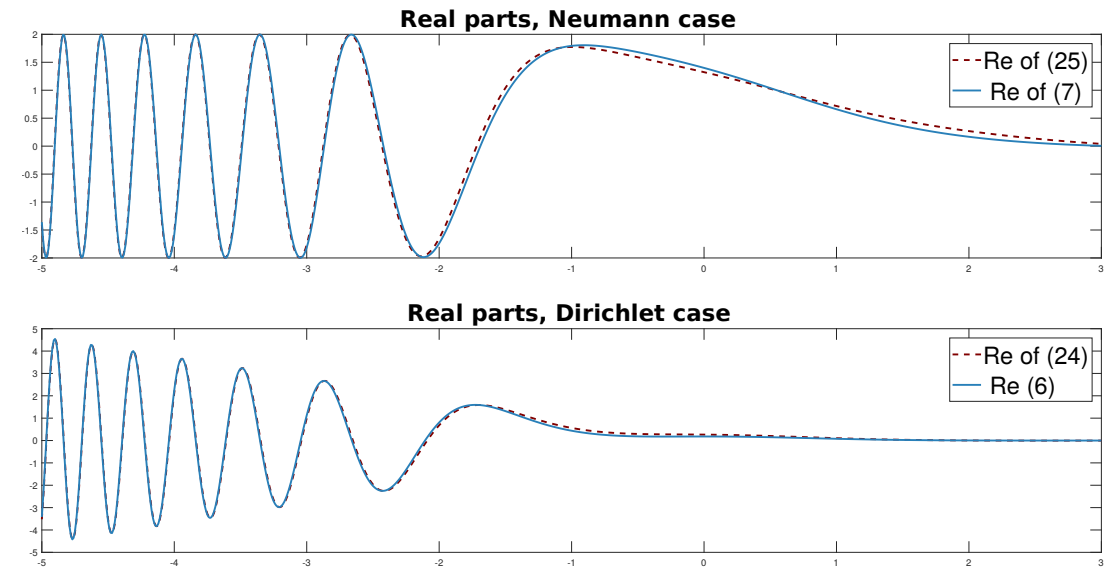

Figure 7: Real parts of Fock's currents (6), (5) are shown with solid line; and the currents with the correction terms (15), (14), are shown with dashed line; here $\varepsilon=1.5, k \rho_{0}=20$, $-5 \leq \sigma \leq 3$.
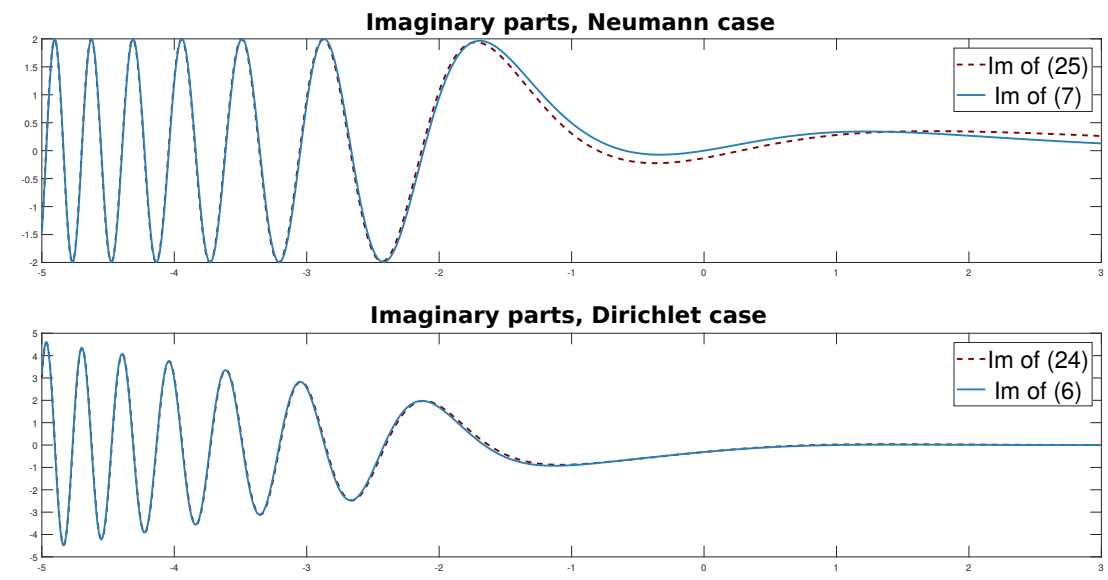

Figure 8: Imaginary parts of Fock's currents (6), (5), are shown with solid line; and the currents with the correction terms (15), (14), are shown with dashed line; here $\varepsilon=1.5$, $k \rho_{0}=20,-5 \leq \sigma \leq 3$. 


\section{Relative influence of the correction terms}

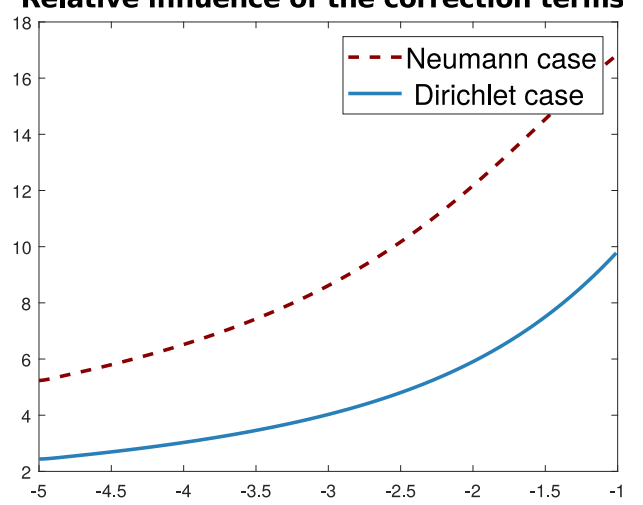

Figure 9: The absolute value of the relative error in percents, for $\varepsilon=0.1,-5 \leq \sigma \leq-1$, $k \rho_{0}=20$.

in the Fock's zone. The relative correction term for the current (see Fig. 9) shows that in the lit zone, where the match of the currents is extremely good, the influence of the correction term in the Neumann case is higher.

\section{Conclusions}

All the scatterers with the smoothly changing curvature have the same currents distribution if their curvature at $s=0$ is the same; and if the incident wave is the same.

The difference between the Dirichlet current (5) and the Neumann current (6) is in the order of their amplitudes: the amplitude is of order $\mathcal{O}\left(\boldsymbol{M}_{0}^{-1}\right)$ compared to the Neumann case.

In the case of Neumann boundary conditions, the wave field penetrates the boundary shadow region 5 deeper compared to the Dirichlet case. The wave field on the limit ray 3 is strongly non-zero in the Neumann case.

The obtained formulae for the currents are valid near the surface of the scatterer, however, they give a way to determine the field at greater distances, using quadratures.

Our future plans are to obtain and evaluate the behaviour of the creeping waves in the shadow zone 5 and compare the influence of the elongation.

\section{ACKNOWLEDGMENTS}

The authors are very grateful to Prof. M. M. Popov and Dr. N. M. Semtchenok for the valuable advice and suggestions regarding the subject of the paper. N. Kirpichnikova has been partially supported by RFBR grant 17-01-00529_A.

\section{REFERENCES}

[1] Kirpichnikova, N. Ya., Popov, M. M., 2013, Leontovich-Fock parabolic equation method in the problems of short-wave diffraction by prolate bodies, Journal of Mathematical Sciences, Vol. 194(42), pp. 30-43.

[2] Popov, M. M., Kirpichnikova, N. Ya., 2014, On problems of applying the parabolic equation to diffraction by prolate bodies, Acoustical Physics, Vol. 60(4), pp. 363-370.

[3] Kirpichnikova, N. Ya., Popov, M. M., 2016, Merging of asymptotics in the illuminated part of the Fock domain, Journal of Mathematical Sciences, Vol. 214(3), pp. 277-286.

[4] Kirpichnikova, A. S., Kirpichnikova, N. Ya., 2017, The Leontovich-Fock parabolic equation method in the Neumann diffraction problem on a prolate body of revolution, Zapiski Nauchnykh Seminarov POMI, Vol. 461(47), pp. 148-173.

[5] Popov, M. M., Semtchenok, N. M., Kirpichnikova, N. Ya., 2017, On short-wave diffraction by an elongated body. Numerical experiments, Journal of Mathematical Sciences, Vol. 226(6), pp. $734-743$.

[6] Fock, V.A., 1965, Electromagnetic Diffraction and Propagation Problems, International Series of Monograph on Electromagnetic Waves, Vol. 1, Pergamum Press, Oxford, London, Edinburgh, New York, Paris, Frankfurt.

[7] Babich, V. M., Kirpichnikova, N. Y., 1979, The Boundary-Layer Method in Diffraction Problems, Springer-Verlag, Berlin, Heidelberg, New York. 